Review

\title{
Review on the State of Charge Estimation Methods for Electric Vehicle Battery
}

\author{
Mingyue Zhang * $\mathbb{D}$ and Xiaobin Fan $\mathbb{D}$ \\ School of Mechanical and Power Engineering, Henan Polytechnic University, 2001 Century Avenue, \\ Jiaozuo 454003, China; fanxiaobin@hpu.edu.cn \\ * Correspondence: 15738515780@163.com
}

Received: 9 January 2020; Accepted: 10 March 2020; Published: 11 March 2020

\begin{abstract}
Battery technology has been one of the bottlenecks in electric cars. Whether it is in theory or in practice, the research on battery management is extremely important, especially for battery state-of-charge estimation. In fact, the battery has a strong time-varying and non-linear properties, which are extremely complex. Therefore, accurately estimating the state of charge is a challenging task. This paper reviews various representative patents and papers related to the state of charge estimation methods for an electric vehicle battery. According to their theoretical and experimental characteristics, the estimation methods were classified into three groups: the traditional methods based on the battery experiments, the modern methods based on control theory, and other methods based on the innovative ideas, especially focusing on the algorithms based on control theory. The results imply that the algorithms based on control theory, especially intelligent algorithms, are the focus of research in this field. The future development direction is to establish a rich database, improve hardware technology, come up with a much better battery model, and give full play to the advantages of each algorithm.
\end{abstract}

Keywords: batteries; control-theory-driven estimation; electric vehicles; multi-scale; state of charge; test-driven estimation

\section{Introduction}

When it comes to environmental pollution, many people will associate it with cars. Indeed, traditional fuel cars have brought these problems such as the greenhouse effect and haze. As a result, the focus of people is again turning toward new energy vehicles, especially electric-powered vehicles, which will be the new trend in the future [1]. At present, all countries in the world are involved in electric-powered vehicles research and have highlighted that battery management is one of the bottlenecks in the development of electric-powered vehicles. Developed countries like the United States, Germany and Japan have introduced corresponding policies to improve the research on battery [2]. At the same time, China has also passed the Tenth Five-Year Plan, Eleventh Five-Year Plan, and Twelfth Five-Year Plan to make China's electric vehicle industry progress.

Efficient battery management of electric vehicles plays an important role in ensuring vehicle safety, extending battery life, reducing cost and improving driving range [3-5]. A common Battery Management System (BMS) in the actual vehicle is mainly composed of a variety of sensors, actuators, controllers and signal lines, as shown in Figure 1. More BMSs have been installed the equalization management, fault diagnosis and so forth. Among them, the main task of the sampling circuit is to measure voltage, current, and temperature signals. Then the control circuit uses these signals for the estimation of the State of Charge (SOC), State of Health (SOH), State of Power (SOP) and State of Life (SOL) of batteries, making use of all kinds of algorithms available. Eventually, the vehicle's 
controller deals with such circuits and realizes the management of the automobile energy and power distribution [6-9].

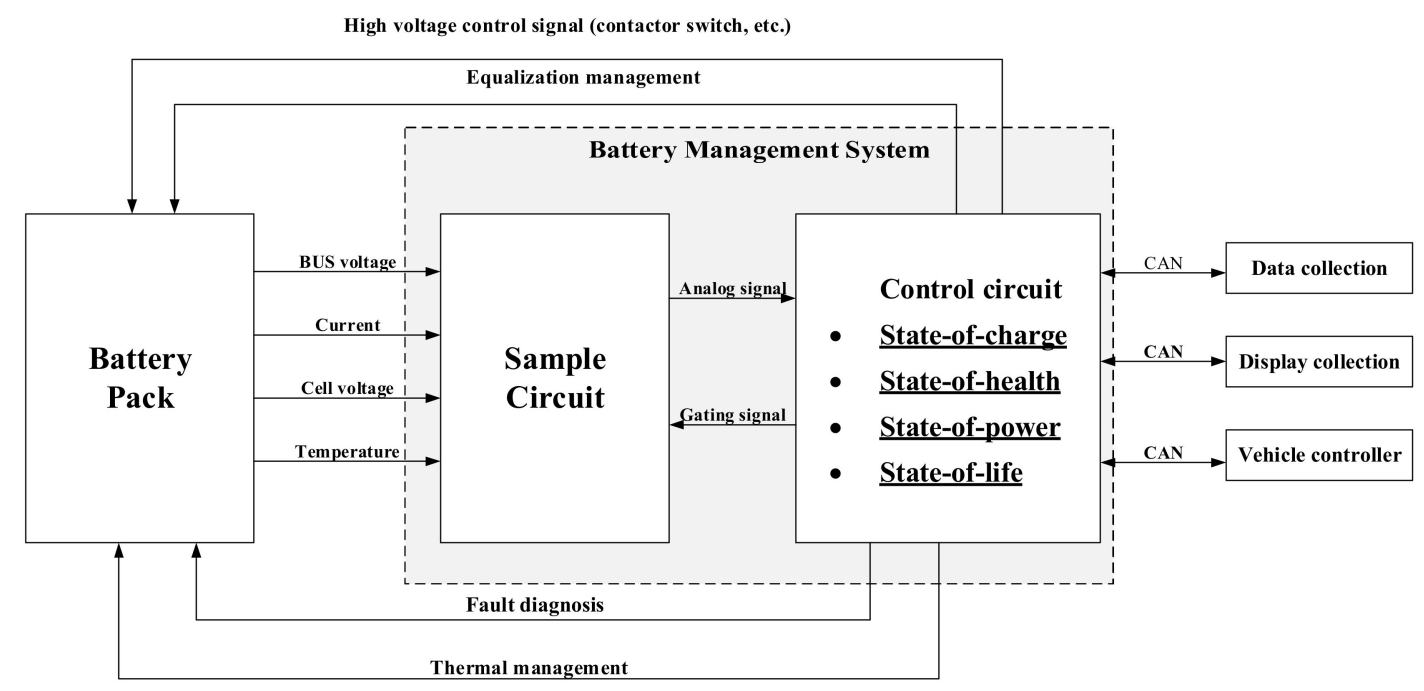

Figure 1. The general function of a battery management system.

The state of charge of batteries in a battery management system is like the fuel meter in a conventional fuel car. The main function of the SOC is to communicate the instinctive battery state to the driver and at the same time, avoid problems such as overcharge and over-discharge [10,11]. In fact, the estimation of the SOC has been a problem under study. As we know, the battery is a very complex and non-linear electrochemical element, and its performance is relative to its internal and external conditions. At the same time, the battery's performance should not only consider the performance of the single battery but also consider the inconsistency of battery performance in the battery pack. In addition, battery aging, cycle life, temperature, and other factors will bring great challenges to the estimation of the charged state of the battery [12-14]. In fact, scholars and researchers began to study SOC in the 1960s. Over the past half-century, there has been a lot of scientific research works on the SOC estimation of batteries, but this problem still needs a more effective solution [15-18]. Therefore, this paper studies the existing SOC estimation methods, to make it helpful for scholars, researchers, and automobile enterprises.

The structure of this paper is as follows: Section 2 introduces the concept of SOC. Section 3 classifies SOC estimation methods and analyzes their advantages and disadvantages, respectively. Section 4 draws a conclusion and gives suggestions.

\section{The Definition of SOC}

The SOC of the battery refers to the ratio of the current remaining battery capacity to the available capacity under certain conditions (temperature, charge and discharge ratio, etc.), and its mathematical expression is shown in Equation (1).

$$
S O C=\frac{Q_{c}}{Q} \times 100 \%=100 \%-\frac{Q_{e}}{Q} .
$$

However, different scholars hold different views on this issue [19]. The difficulty lies in the understanding of the numerator and the equation's denominator, and the denominator of Equation (1) is the battery capacity. The definition of the battery capacity known here is not consistent. The rated capacity, factory capacity, cycle capacity, or current battery actual capacity is usually used as the denominator of Equation (1) [20-22]. In the theoretical analysis, the most used capacity is rated capacity as a classic definition of the denominator of Equation (1). This method regards the rated capacity as a fixed value, and the SOC is obtained by subtracting the amount of charge or discharged from the rated 
capacity [23]. At present, most electric vehicles define SOC from the perspective of electric charge quantity, so in this equation, $Q_{c}$ is the residual power of the battery at the moment of calculation, and its unit is A.h; $Q$ is the total capacity of the battery, and its unit is A.h [24]. $Q_{e}$ is the battery charge.

In fact, the battery usually varies with many factors, and this equation needs to be modified [25]. The Equation (2) is more commonly used.

$$
\operatorname{SOC}(t)=S O C t_{0}-\int_{t_{0}}^{t} \frac{\eta I}{C_{n}} d \tau
$$

In this equation, $\operatorname{SOC}(t)$ is the nominal capacity of the battery, and its unit is A. $h \cdot \eta$ is the coulomb efficiency, also called the discharged efficiency, which refers to the ratio of the discharge capacity of a battery to the charge capacity in one $\operatorname{loop}\left(\eta=Q / Q_{n}\right)$. The entered charge is often unable to convert all the active substances into electricity, because there is a certain loss, such as the battery occurred irreversible side reactions. Therefore, the value of $\eta$ is usually less than $100 \%$. In fact, the current lithium-ion batteries have a coulomb efficiency of $99.9 \%$ or more. Its value can be obtained by the Peukert equation, combining the measured residual charge and discharge current of the two batteries. However, in practice, it is difficult to measure the coulomb efficiency, which is extremely susceptible to the influence of charge and discharge current, temperature, battery aging degree and internal resistance of the battery [26].

When talking about SOC, another parameter to consider is the state of health $(\mathrm{SOH})$. $\mathrm{SOH}$ can well reflect the aging degree of battery. $\mathrm{SOH}$ is affected by various kinds of factors. During the use of battery assembly vehicle, due to the influence of temperature, ventilation condition, self-discharge degree, electrolyte concentration and other differences of the batteries in the battery pack, the inconsistencies of battery voltage, internal resistance, capacity and other parameters will be increased to some extent, which will affect the value of $\mathrm{SOH}$. The relationship between the two is as follows.

$$
\operatorname{SOC}(t)=S O H(t)-D O D(t)
$$

In Equation (3), $\mathrm{SOH}(t)$ is the state of charge. When the battery is a new one, we consider $\mathrm{SOH}$ as $100 \%$. $D O D(t)$ (depth of discharge) represents the percentage of discharge of the battery and the rated capacity of the battery. DOD is considered when the discharge of the battery exceeds at least $80 \%$ of its rated capacity.

\section{SOC Estimation Methods}

SOC is an important part of BMS. Based on the theoretical and experimental characteristics, various estimation methods are classified into three groups: the traditional estimation algorithm based on experiments, modern methods based on control theory and other methods based on the innovative ideas. As shown in Figure 2, we classify these methods into three groups to compare them in more detail. 


\section{SOC estimation methods}
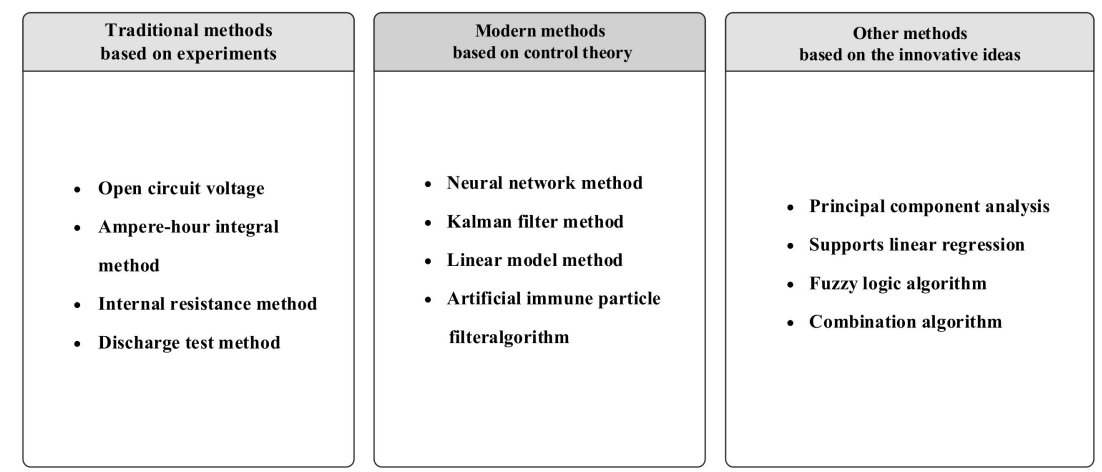

Figure 2. Classification of the State of Charge (SOC) estimation methods.

\subsection{Traditional Methods Based on Experiments}

\subsubsection{Open Circuit Voltage}

The Open Circuit Voltage method, also known as the Voltage measurement method, is based on the corresponding relationship between Open Circuit Voltage (OCV) and SOC in Equation (4).

$$
U o c=f(S O C)
$$

In this correspondence, the experimental methods help us obtain $U_{o c}$ and $f(s o c)$ can be obtained through the charge and discharge fitting experiment of the battery [27].

When determining the above relationship, it is necessary to establish the PNGV model and refer to the PNGV battery experiment manual for the HPPC experiment to obtain the SOC-OCV relationship curve. As shown in Figure 3, the PNGV model adds the capacitance $C_{P}$, compared with the Thevenin model. The advantage of the PNGV model is that it need not high-performance processor and is easy to implement, so it is suitable for simulation dynamic analysis. The disadvantage of the PNGV model is that it does not consider the charging process, so it is not suitable for long-term stable discharge simulation of the battery.

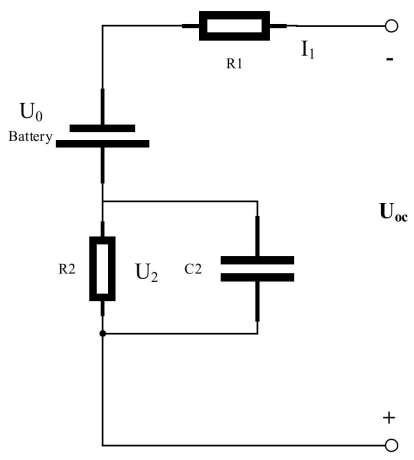

(a) Thevenin model

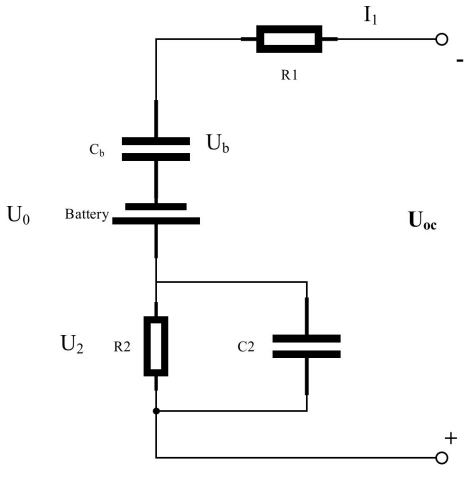

(b) PNGV model

Figure 3. Battery model.

As shown in Figure 4, the corresponding relationship is shown between OCV and SOC of lithium iron phosphate battery and lithium manganese acid battery. 


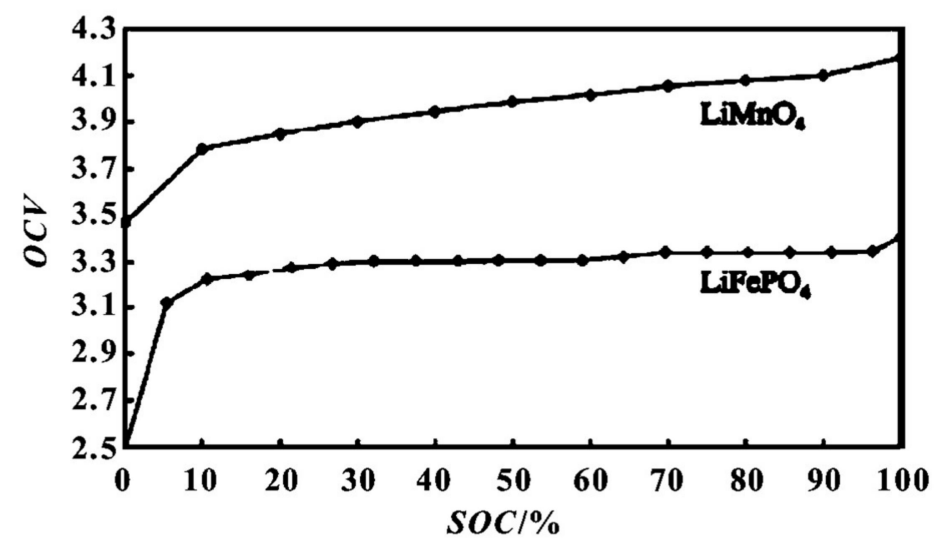

Figure 4. Open Circuit Voltage (OCV) curve of lithium iron phosphate and lithium manganese acid battery.

The advantage of the OCV method is that the measured quantity is relatively simple, and the accuracy obtained is higher [28-32]. Moreover, its shortcomings are also very obvious, for measuring the terminal voltage, it needs to be placed for a long period of time (a few hours or even longer), so the amplifier cannot be used for continuous dynamic on-line estimation. At the same time, because there is a platform phenomenon in the battery voltage, which means paying more efforts on the current hardware technology to guarantee accuracy requirements. Therefore, the open-circuit voltage method is always together with other methods at the initial or final stage of charge, and rarely used alone, such as the SOC estimation method combining the open-circuit voltage method with the ampere-hour integral method $[33,34]$, and the SOC estimation method combining the open-circuit voltage method with the Kalman filtering method [35].

\subsubsection{Ampere-Hour Integral Method}

The Ampere-hour (Ah) integral method is the most commonly used method for electric charge accumulation at present. It is based on the calculation of electric quantity during charging and discharging. In fact, we also take the temperature, charging and discharging efficiency of the battery into account, when estimating SOC [36-41]. Equation (5) can illustrate the Ah integral method.

$$
S O C=\frac{Q_{0}+\int_{0}^{t} i_{c} \eta d t-\int_{0}^{t} i_{d} d t-S}{Q}
$$

In Equation (5), $Q$ is the rated capacity of the battery, unit: A. $h . Q_{0}$ is the initial charge of the battery, unit: A.h. $\eta$ is the charging efficiency. $S$ is the electric quantity of self-discharge, unit: A.h. $i_{c}$ is the charging current, unit: A. $i_{d}$ is the discharge current, unit: A.

Bao hui et al. proposed an improved Ah integral method to correct the estimated error value. The principle is shown in Equation (6).

$$
S O C=\alpha S O C_{0}-\frac{1}{\delta c} \int_{0}^{t} \eta_{\varepsilon} I d t .
$$

In this equation, $S O C_{0}$ is obtained by OCV method. $\alpha$ is the self - discharge rate and aging factor correction factor, which can be obtained by plenty of experiments. $\delta$ is correction factor for battery capacity (c), which can be obtained by this equation $\left(\delta=0.0010 \mathrm{~N}^{2}-0.032 \mathrm{~N}+11.8819\right)$. $\mathrm{N}$ is the number of cycles. The parameter $\eta_{\varepsilon}$ is the equivalent coulomb efficiency. Its value can be obtained by unifying the coulomb efficiency of different currents to $5 \mathrm{~h}$ multiplier discharge current $\left(I_{5}\right)$. The experimental results show that it is robust and accurate.

The Ah integral method has the advantages of simple calculation, stable algorithm and online measurement. Considering the battery's self-discharge, temperature, charging and discharging 
efficiency of the battery, the method can meet certain accuracy requirements in the early use of the battery. Every coin has two sides, so the disadvantage of this method is also obvious. First, because the quantity needs to be measured is so large that untimely, there is always a chance of errors. Second, the battery will be aged in use, and the loss capacity cannot be compensated. Moreover, the battery's discharge capacity in the process can be recovered, but the charging capacity is not easy to eliminate the effect. Finally, the measurement error will accumulate with time going by.

At present, many researchers try to come up with new methods, based on the ampere-hour integral method to improve the accuracy of SOC, for example, merging the Ah integral method together with the OCV method, and using OCV to modify the initial SOC to improve the estimation accuracy $[42,43]$.

\subsubsection{Internal Resistance Method}

The internal resistance method aims to obtain the SOC value according to the relationship between the internal resistance of the battery and SOC [44-46]. The relationship between the internal resistance of the battery and the SOC is based on the relationship between internal resistance and voltage change $[47,48]$. Taking the PNGV model in Figure 3 as an example, the following relationship can be obtained. There is a basic principle in Equation (7).

$$
U_{o c}=U_{0}-I_{1}\left(R_{1}+R_{2}\right)-U_{b}
$$

In Equation (7), $U_{o c}$ is the open-circuit voltage of the battery. $R_{1}$ is the ohmic resistance of the battery. $R_{2}$ is the polarization resistance of the battery, which forms a parallel RC loop with the polarization capacitor $C_{2}$. The polarization effect inside the battery can be simulated. $C_{b}$ is the battery capacitor, which is due to the OCV change blocked together with the current $I_{1}$.

Theoretically, the internal resistance method is relatively simple, mainly because it only considers the discharge current and internal resistance of the battery. However, in practice, the relationship between battery parameters and SOC is quite complex. At the initial stage of discharge, the discharge efficiency of the internal resistance is stable and almost has no fluctuation. However, at the later stage of discharge, the internal resistance will obviously increase and will show obvious fluctuations. Therefore, the internal resistance method is always used for SOC estimation in the later stage of discharge.

As we all know, the internal resistance of the battery can be divided into the AC internal resistance (also known as AC impedance) and the DC internal resistance. Thus, the internal resistance method can also be divided into two methods. The AC impedance is actually a transfer function between the voltage and the current of the battery. It is usually measured by an AC impedance meter. However, due to the great influence of temperature, it has always been controversial whether it should be estimated in the open-circuit state or in the process of charging and discharging. Therefore, this method is rarely used in real cars.

In fact, SOC estimation usually uses DC internal resistance as an object. Nevertheless, this method also has its limitations. For example, the relationship between the internal resistance and SOC of a nickel-hydrogen battery and a lithium-ion battery is more complicated than that of a lead-acid battery, so the internal resistance method is not applied to the SOC estimation of a lithium-ion battery $[48,49]$. Now, most modern automobiles use lithium-ion batteries as power batteries, so the internal resistance method is also difficult to apply to electric vehicles. The above has been adjusted and supplemented in the text and highlighted.

The internal resistance is the ratio of the voltage change to the current change over a short period of time. This result is often affected by the calculation time. The internal resistance will become more complex when the time is too long, and it will change with different working stages. Therefore, it is quite difficult to estimate SOC value by the internal resistance method, which is not often used alone. It is usually combined with the Ah integral method to estimate SOC in the later stage of discharge. 


\subsubsection{Discharge Test Method}

The discharge test method is the most reliable SOC estimation method at present $[33,37,50]$. Its principle is that in the constant current continuous discharge, the SOC equals that the discharge current multiples time. Its advantage is that it has good applicability for almost all batteries. It is mainly used in the laboratory as the standard of SOC accuracy, as well as in the research on charging and discharging characteristics and battery maintenance. However, the whole experimental process needs a lot of time, so the SOC measurement with the vehicle in motion cannot be carried out.

In summary, the traditional estimation method based on the experiment has the advantage that the algorithm is simple and easy to implement, but the higher requirements of the hardware require a large amount of experimental investment and it only has a significant performance in estimating SOC. With respect to the estimation accuracy and test characteristics of each method, the accuracy of the open-circuit voltage method increases with the battery's stand by time, which means that the test time will be extended accordingly. The Ampere-hour (Ah) integral method is mainly used for the initial state-of-charge estimation, but its accuracy decreases with the test time, because the internal resistance constantly changes due to many factors. The internal resistance method is stable only in the late stage of discharge and has a high degree of precision, but the test time is often not accurately obtained which will cause an error. The discharge test method has high precision, but it is not suitable for real vehicles and can only be performed in the laboratory stage.

\subsection{Modern Methods Based on Control Theory}

\subsubsection{Neural Network Method}

The neural network is a branch of artificial intelligence. It bases on a simple abstraction and simulation of the human brain and accepts the corresponding training mainly through the input and output sample so that it can meet the mapping function relations. It improves the accuracy of the model through the model weight and deviation adjustment $[51,52]$. This process can be divided into two stages.

The first is the positive calculation process, which mainly completes the calculation of each unit from the input layer to the output layer.

The second is the process of error echo propagation.

In Figure 5, the input layer is mainly the battery performance parameters such as current, voltage, temperature and internal resistance. The output layer is the estimated value of the battery's SOC. The hidden layer is the activation function of the system. According to the research of Xia Kegang et al., the basic processing process of neural network algorithm is shown in Figure 6. The experimental results show that the method is robust and accurate.

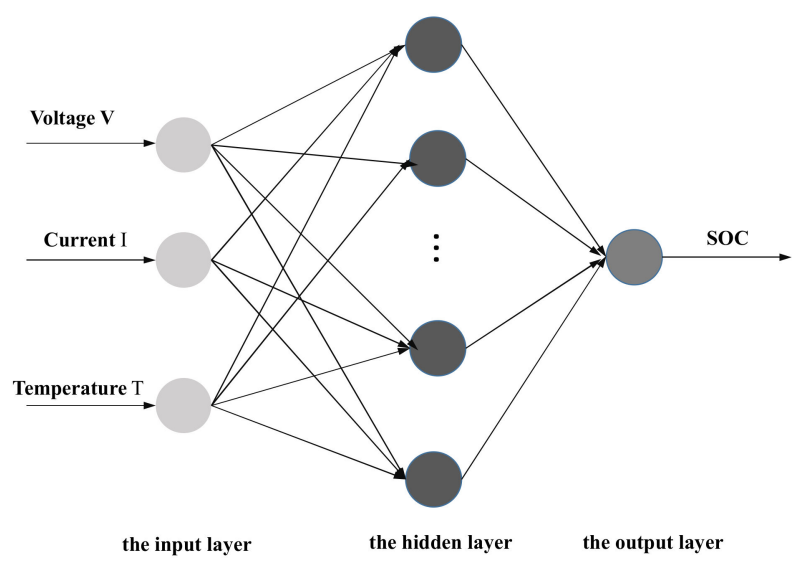

Figure 5. SOC estimation principle of neural network. 


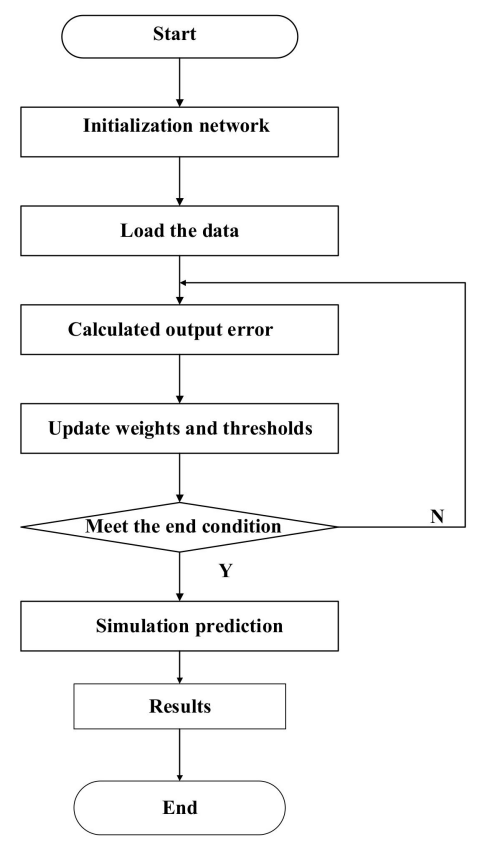

Figure 6. Process of neural network.

The advantages of this method lie in that it can accurately estimate the SOC quickly and easily. At the same time, the parallel and global searching methods are fast and it has better convergence speed and precision in the experiment. The disadvantages of this method are also obvious, mainly because it requires a large amount of training data as a support to complete the training system. Training data and training methods have a great impact on its accuracy $[48,53,54]$. In practice, it also needs further study, because its algorithm is too complex and needs a large amount of calculation [55-57].

\subsubsection{Kalman Filter Method}

The core idea of the Kalman Filter (KF) is the optimal design of the least variance of the state of the power system [58-60]. The KF method for SOC estimation treats the battery as a power system.

The general mathematical form of the battery model is as follows.

Equation of state:

$$
x_{k+1}=f\left(x_{k}, u_{k}\right)+w_{k}
$$

Equation of observation:

$$
y_{k+1}=g\left(x_{k}, u_{k}\right)+v_{k} .
$$

In Equation (8) and (9), $u_{k}$ is the input of the system, generally referring to the variables such as current, temperature, residual electric quantity, and internal resistance. $y_{k+1}$ is the output of the system, usually referring to the voltage, $x_{k}$ is the state quantity of the system, including the estimated value of SOC. Functions $f\left(x_{k}, u_{k}\right)$ and $g\left(x_{k}, u_{k}\right)$ refer to the nonlinear equations established on the battery model, but they need to be linearized in the calculation process.

The principle of this method is shown in Figure 7. It describes the battery as a system composed of an equation of state and observation equation, considers SOC as an internal state of the system, establishes a state-space model, and makes the minimum variance estimation for SOC.

The Kalman Filter algorithm needs to base on the specific equivalent circuit model to establish the corresponding equation of state. Qian Xiaoxiao etc. uses the PNGV model to establish the battery 
state equation, as is shown in the Equation (10) and (11), and Kalman Filter algorithm to calculate its coefficient matrix.

$$
\begin{aligned}
& {\left[\begin{array}{c}
\dot{U}_{b} \\
\dot{U}_{2}
\end{array}\right]=\left[\begin{array}{cc}
0 & 0 \\
0 & -\frac{1}{\mathrm{R}_{2} C_{2}}
\end{array}\right]\left[\begin{array}{l}
U_{b} \\
U_{2}
\end{array}\right]+\left[\begin{array}{c}
\frac{1}{C_{b}} \\
\frac{1}{C_{2}}
\end{array}\right] I} \\
& U_{o c}=\left[\begin{array}{ll}
-1 & -1
\end{array}\right]\left[\begin{array}{l}
U_{b} \\
U_{2}
\end{array}\right]+\left(-R_{1} I\right)+U_{o c} .
\end{aligned}
$$

Its advantage is that it eliminates the error of the ampere-hour integral method accumulated over time. At the same time, it does not have a high requirement for the accuracy of the initial SOC. In other words, even if the initial value has a certain deviation it can well converge to the real value. Even if there is noise, it can have a good correction effect.

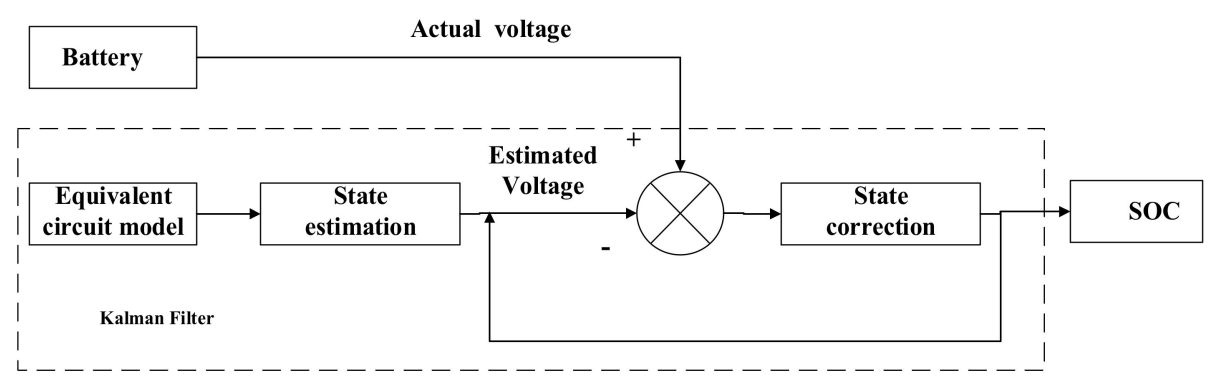

Figure 7. The principle of Kalman Filter (KF).

Its disadvantage is that the accuracy largely depends on the establishment of the battery equivalent model, and the error mainly comes from three aspects: the time variability of the model, the non-linearity of the model and the approximate treatment of noise. In practical applications, it is actually used in a variety of batteries, especially in hybrid-electric vehicles with violent current fluctuations.

At present, many improved methods have been generated based on this method [61-66]. For example, the Extended Kalman Filtering (EKF) method, which linearizes the nonlinear system; the Unscented Kalman Filtering (UKF) method is obtained by adding the transformation of U to KF which deals with the nonlinear problem with a probability distribution; the Central Difference Kalman Filtering (CDKF) method processes KF using the central difference method., etc.

\subsubsection{Linear Model Method}

The linear model method is a linear equation based on variations in the last moment, such as current, voltage and SOC value [67-69].

The equation is shown below:

$$
\begin{gathered}
\triangle S O C(i)=\beta_{0}+\beta_{1} U(i)+\beta_{2} I(i)+\beta_{3} \operatorname{SOC}(i-1) \\
\operatorname{SOC}(i)=\operatorname{SOC}(i-1)+\Delta \operatorname{SOC}(i) .
\end{gathered}
$$

In Equation (12) and (13), $S O C(i)$ is the SOC value now. $\triangle S O C(i)$ is the change quantity of SOC; $U(i)$ is its voltage; $I(i)$ is current; $\beta_{0}, \beta_{1}, \beta_{2}$ and $\beta_{3}$ are the coefficients obtained by the least square method with reference data.

The model above is established on the basis of experiments. This method is mainly applicable to low current and slow change of SOC and is also applicable to batteries at all stages. However, as far as the current situation is concerned, it does not have universality. Thus, further research on the applicability of other batteries needs to be carried out. 


\subsubsection{Particle Filter Algorithm}

The idea of the Particle Filter (PF) is based on Monte Carlo methods, which use particle sets to represent probabilities and can be used in any form of the state space model. The core idea is to express its distribution by extracting random state particles from the posterior probability. It is also a sequential importance sampling. In simple terms, the particle filter method refers to the process of finding the state's minimum variance distribution by finding a set of probability density functions of a set of random samples propagating in the state space, then approximating them, and using the sample mean instead of the integral operation. The sample here is the particle, and when the number of samples $(N)$ is near infinity, it can approximate any form of probability density distribution. However, this means that in order to get high precision, a large number of samples are needed for support. The PF is an optimal filtering technique for estimating non-Gaussian nonlinear systems without any restrictions on process noise and observed noise [70].

The commonly used particle filter algorithms for SOC estimation are Artificial Immune Particle Filtering (AIPF) algorithms and the improved particle filter which is obtained by combining particle filtering algorithms with other estimation methods, such as the Ah integration method, Kalman filtering algorithm and so on [71-73]. Next, the AIPF is taken as an example to analyze the principle and characteristics of the particle filter algorithm.

The AIPF is based on a series of weighted particles sampled by the Kurt Russell method to approximate the posterior distribution of the system, without any explicit assumptions about the distribution, which are not available in the KF method and the neural network method [74-76]. The AIPF requires the establishment of distribution approximate to the posterior distribution, which is actually quite difficult $[73,77]$.

The principle of AIPF can be described as in Figure 8. In Figure 8, the importance of density function is based on random $N$ samples, which is illustrated as the initial antibody. The $N$ antibody is the particle of state estimation. Then process the $N$ particle, respectively calculate the affinity and non-affinity among $N$. According to the size of the affinity, determine how many each antibody should be cloned. The new antibody cluster was composed of $N$ initial antibodies and the antibodies obtained after cloning. Their affinity and non-affinity were calculated again; then similar antibodies were discarded, and the best $N$ antibodies were selected from the antibody group as the estimation of the state at the next moment, and the optimal particles can be acquired.

The advantage of this approach is that it can be able to make these particles in the optimal state. The shortcomings are quite tedious in the calculation of the whole process.

In summary, the estimation method based on modern control theory has high precision and can deal well with the influence of accumulated error and noise on estimation accuracy, but its algorithm is relatively complex and has high requirements on the accuracy of the battery model. From the perspective of each estimation method, the higher response speed and higher precision of the neural network algorithm is obvious, but it requires a large number of experiments as support; the accuracy of the Kalman Filter algorithm depends most on the battery model, and the testing time influences less on its accurate. Although the linear model estimation method is suitable for estimating the state of charge of the battery, the type of battery used for trial is less; the particle filter algorithm can obtain higher precision, but the algorithm is complex and the testing time is longer. 


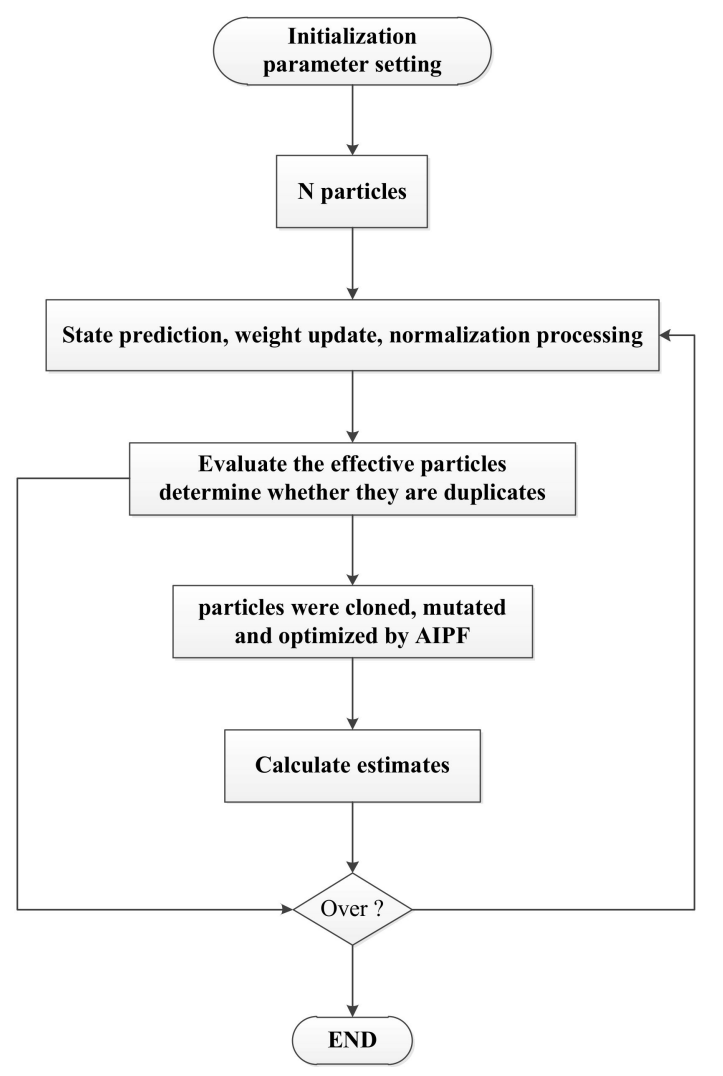

Figure 8. The principle of the Artificial Immune Particle Filtering (AIPF) algorithm.

\subsection{Other Methods Based on the Innovative Ideas}

With the progress of research, there are more and more SOC estimation algorithms. They have higher accuracy and stronger adaptability. Other common SOC algorithms are as follows, which are often based on innovative ideas. They are often derived from knowledge in other subject areas or a combination of methods to take advantage of the various methods [78-88].

Principal Component Analysis (PCA) is an algorithm based on multivariate statistics, which can analyze and process data by simplifying and compressing various data and extracting important elements. The main methods used are zero mean method and least square method.

However, this method is not suitable for different temperatures and different degrees of battery deterioration. On the basis of experiments, Hao Zhonghua etc. obtained the main factors affecting SOC performance (resistance, voltage, current) as the object, then used the least square method to deal with the main factors affecting SOC performance and through the model simulation to test the feasibility of the method. The experimental results show that this method can effectively improve the PCA and is not suitable for temperature variability. It's different from battery degradation. The mean fault error is improved well.

The basic idea of Supports Vector Regression (also known as SVR) is based on the idea of minimizing structure empirical risk and model complexity. Compared with the neural network method, it has better practicability and it is easier to approach the real value of SOC. It is very important to select kernel function in SVR, and proper kernel function can make this method have better generalization ability. In general, the Gaussian radial basis function is selected as the sum function to obtain a simple nonlinear mapping with fast convergence speed and few parameters. Zhu Jiang et al. took voltage and temperature as inputs and SOC as outputs, and compared it with the neural network algorithm, and found that the SVR method had higher prediction accuracy and stability.

In addition, some researchers combine no less than two algorithms, so as to make full use of the advantages of each algorithm, resulting in more accurate and more adaptive algorithms. 
Deng Tao et al. obtained a new estimation method by combining Ah integral method, open-circuit voltage method and load voltage method. As is shown in the Equation (14), the initial value $\left(S O C_{0}\right)$ is obtained by open-circuit voltage method, and the $S O C_{1}(t)$ was obtained by Ah integration method and the $S O C_{2}(t)$ corresponding to load voltage method are averaged.

$$
\operatorname{SOC}(t)=S_{0}-S_{0}(t)+S_{1} C_{2}(t) .
$$

Wu proposed a kind of SOC estimation method based on neural networks and the Master-Slave Adaptive Unscented Kalman Filter (MS-AUKF) algorithm. LIU Xin-tian proposed that the Drift-Ah integral method is proposed with drift current, the noise combination model was established, and the SOC estimation of lithium battery was achieved by using the Cubature Kalman Filter (CKF).

These estimation methods will be continuously updated and improved. From the current situation, the accuracy is constantly improving, and the response speed is also increasing.

\section{Conclusions}

In the field of electric vehicles, lithium batteries have become a research hotspot by virtue of their own advantages. SOC estimation, as a very important and challenging part of the battery management system, will remain a research hotspot in the future. This review discusses the current SOC estimation in combination with specific research, and classifies the existing methods, which is of great significance to future research.

It can be seen from the discussion above that there are many methods for SOC estimation, and each method has its advantages and disadvantages. There is a comparison of all estimations in Table 1.

Table 1. The comparison of all estimations and their advantages and disadvantages.

\begin{tabular}{|c|c|c|}
\hline Estimation & Advantages & Disadvantages \\
\hline $\begin{array}{l}\text { Traditional methods } \\
\text { based on experiments }\end{array}$ & $\begin{array}{l}\text { The simple and stable algorithm is simple and stable } \\
\text { Mature technology } \\
\text { Easy to implement }\end{array}$ & $\begin{array}{c}\text { High requirements on hardware } \\
\text { The effect is usually better in a certain } \\
\text { period of battery estimation } \\
\text { A large amount of experimental } \\
\text { investment is required } \\
\text { Obvious cumulative effect of errors }\end{array}$ \\
\hline $\begin{array}{l}\text { Modern methods based } \\
\text { on control theory }\end{array}$ & $\begin{array}{l}\text { Better eliminate error accumulation effect } \\
\text { Correct the noise well } \\
\text { High convergence speed and accuracy }\end{array}$ & $\begin{array}{l}\text { Higher requirements for battery model } \\
\text { The algorithm is too complex }\end{array}$ \\
\hline other methods & $\begin{array}{l}\text { Strong pertinence } \\
\text { Interdisciplinary and interdisciplinary applications }\end{array}$ & $\begin{array}{l}\text { The practical applicability needs to be } \\
\text { further verified } \\
\text { Complex algorithm }\end{array}$ \\
\hline
\end{tabular}

From Table 1, we can know that in the current SOC estimation methods, the traditional algorithm is the simplest and the most widely used algorithm, but its accuracy needs to be improved; the modern method has high accuracy, which is the current research hotspot, but it is limited by the current hardware technology; there are many other methods, and most of them are combined algorithms.

In practice, the internal resistance method and the OCV method are usually not used alone but combined with the Ah integral method to make full use of their advantages. The KF series algorithm realizes the on-line estimation of SOC and the accurate estimation of the nonlinear system. The particle filter series algorithm can improve the SOC estimation accuracy on the basis of the KF series algorithm, but its operational complexity is high. The improved trackless particle filter algorithm also effectively reduces operational complexity. However, the linear model method, neural network method and other methods still need to be improved, and the same is true for other methods.

\section{Current and Future Developments}

In general, the traditional estimation methods based on experiments are relatively mature, but they are often not used alone due to their limitations. They are often used in combination with other methods and account for the largest proportion in actual use. The estimation method based on modern 
control theory has higher precision and response speed, but the algorithms are obviously complex and require strong theory as support. Their proportion of the actual use process is increasing year by year. The accuracy and response speed of other algorithms based on innovative ideas have been significantly improved and can make full use of the advantages of various methods, but most of them are in the laboratory stage, and they are rarely used in real vehicles.

Taking the above various SOC estimation methods into consideration, it can be found that in the future, traditional estimation methods will still be the mainstream of actual vehicles. The modern estimation method will become a research hotspot, and it needs a lot of experiments and advanced hardware facilities to support its extensive promotion. Other estimation methods will continue to emerge, perhaps again in combination and subject migration.

At the same time, we can find that the following directions will be the focus of their future's works:

1. A rich database should be established to make the SOC estimation more reliable, which depends on a large number of experiments.

2. It is important to improve the hardware technology, improve the accuracy of voltage and current parameters, and strive to ensure the accuracy of SOC.

3. A more accurate battery with good dynamic characteristics and versatility model should be built to accurately describe the dynamic characteristics of the battery in use.

4. We must carry on the effective synthesis of each kind of method, strives for the biggest degree to display respective superiority, promotes the strong point and avoids the weak point.

5. Make full use of interdisciplinary advantages and transfer theoretical knowledge from other disciplines to the remaining electricity estimates.

6. Establish theoretical methods with better dynamic adaptability and precision, and improve the processing methods and theoretical basis of nonlinear systems.

7. Increase efforts to study more stable batteries, such as battery internal resistance and polarization problems.

Author Contributions: Investigation, Mingyue Zhang; writing—original draft preparation, Mingyue Zhang.; writing - review and editing, Xiaobin Fan; funding acquisition, Xiaobin Fan. All authors have read and agreed to the published version of the manuscript.

Funding: This study was supported by Key Scientific and Technological Project of Henan Province (No. 172102210022, No.182102210086), Natural Science Foundation of Henan Province of China (No. 182300410265) and National Undergraduate Training Program for Innovation and Entrepreneurship (No. 201710460096, 201810460069).

Conflicts of Interest: The authors declare no conflict of interest.

\section{References}

1. Bilgin, B.; Magne, P.; Malysz, P.; Yang, Y.; Pantelic, V.; Preindl, M.; Korobkine, A.; Jiang, W.; Lawford, M.; Emadi, A. Making the case for electrified transportation. IEEE Trans. Transp. Electrif. 2015, 1, 4-17. [CrossRef]

2. Hu, X.; Zou, C.; Zhang, C.; Li, Y. Technological developments in batteries: A survey of principal roles, types, and management needs. IEEE Power Energy Mag. 2017, 15, 20-31. [CrossRef]

3. Zhang, C.; Wang, L.Y.; Li, X.; Chen, W.; Yin, G.G.; Jiang, J. Robust and adaptive estimation of state of charge for lithium-ion batteries. IEEE Trans. Ind. Electron. 2015, 62, 4948-4957. [CrossRef]

4. Hu, X.; Li, S.E.; Yang, Y. Advanced machine learning approach for lithium-ion battery state estimation in electric vehicles. IEEE Trans. Transp. Electrif. 2015, 2, 140-149. [CrossRef]

5. Xiong, R.; Zhang, Y.; He, H.; Zhou, X.; Pecht, M.G. A double-scale, particle-filtering, energy state prediction algorithm for lithium-ion batteries. IEEE Trans. Ind. Electron. 2017, 65, 1526-1538. [CrossRef]

6. Yang, R.; Xiong, R.; He, H.; Mu, H.; Wang, C. A novel method on estimating the degradation and state of charge of lithium-ion batteries used for electrical vehicles. Appl. Energy 2017, 207, 336-345. [CrossRef]

7. Castano, S.; Serrano-Jimenez, D.; Sanz, J. BMS influence on Li-ion packs characterization and modeling. In Proceedings of the 2016 IEEE 16th International Conference on Environment and Electrical Engineering (EEEIC), Florence, Italy, 7-10 June 2016. 
8. Hu, X.; Xiong, R.; Egardt, B. Model-based dynamic power assessment of lithium-ion batteries considering different operating conditions. IEEE Trans. Ind. Inform. 2013, 10, 1948-1959. [CrossRef]

9. Peng, S.; Chen, C.; Shi, H.; Yao, Z. State of charge estimation of battery energy storage systems based on adaptive unscented Kalman filter with a noise statistics estimator. IEEE Access 2017, 5, 13202-13212. [CrossRef]

10. Chaoui, H.; Golbon, N.; Hmouz, I.; Souissi, R.; Tahar, S. Lyapunov-based adaptive state of charge and state of health estimation for lithium-ion batteries. IEEE Trans. Ind. Electron. 2014, 62, 1610-1618. [CrossRef]

11. Lu, L.; Han, X.; Li, J.; Hua, J.; Ouyang, M. A review on the key issues for lithium-ion battery management in electric vehicles. J. Power Sources 2013, 226, 272-288. [CrossRef]

12. Bhatt, D.K.; Darieby, M.E. An Assessment of Batteries form Battery Electric Vehicle Perspectives. In Proceedings of the 2018 IEEE International Conference on Smart Energy Grid Engineering (SEGE), Oshawa, ON, Canada, 11-13 August 2018.

13. Sada, T. Battery Diagnosis Method, Battery Diagnosis Program, Battery Management Apparatus, and Power Storage System. U.S. Patent 20190170831A1, 6 June 2019.

14. Meng, J.; Luo, G.; Breaz, E.; Gao, F. A robust battery state-of-charge estimation method for embedded hybrid energy system. In Proceedings of the IECON 2015-41st Annual Conference of the IEEE Industrial Electronics Society, Okohama, Japan, 9-12 November 2015.

15. Sun, F.; Xiong, R.; He, H. A systematic state-of-charge estimation framework for multi-cell battery pack in electric vehicles using bias correction technique. Appl. Energy 2016, 162, 1399-1409. [CrossRef]

16. Marcial-Simon, E.; Mehlhorn, R. Method for Controlling Electrical Charging of a Group of Vehicles. U.S. Patent 20190168633A1, 6 June 2019.

17. VanBlon, R.S.; Peterson, N.J.; Mese, J.C.; Weksler, A.S. Systems and methods to determine time at which battery is to be charged. U.S. Patent 20190148960A1, 16 May 2019.

18. Cai, C.; Du, D.; Liu, Z.; Ge, J. State-of-charge (SOC) estimation of high power Ni-MH rechargeable battery with artificial neural network. In Proceedings of the 9th International Conference on Neural Information Processing, Singapore, 18-22 November 2002.

19. Lillehei, C.W.; Cruz, A.B.; Johnsrude, I.; Sellers, R.D. A new method of assessing the state of charge of implanted cardiac pacemaker batteries. Am. J. Cardiol. 1965, 16, 717-721. [CrossRef]

20. Dincer, I.; Hamut, H.S.; Javani, N. Thermal Management of Electric Vehicle Battery Systems; John Wiley \& Sons: Hoboken, NJ, USA, 2016.

21. Andrea, D.; Li, J. Battery Management Systems for Large Lit-Hium-Lon Battery Packs, 1st ed.; Mechanical Industry Press: Beijing, China, 2016.

22. Rahn, C.D.; Wang, C.-Y. Battery Systems Engineering; Mechanical Industry Press: Beijing, China, 2018.

23. Xiaojun, T. Electric vehicle Battery Management System Design; Sun Yat-sen University Press: Guangzhou, China, 2011.

24. Fang, W.; Jun, X. Design and Manufacture of Battery Management System for Electric Vehicle, 2nd ed.; Science Press: Beijing, China, 2017.

25. Plett, G.L. Extended Kalman filtering for battery management systems of LiPB-based HEV battery packs: Part 3. State and parameter estimation. J. Power Sources 2004, 134, 277-292. [CrossRef]

26. Gholizadeh, M.; Salmasi, F.R. Estimation of state of charge, unknown nonlinearities, and state of health of a lithium-ion battery based on a comprehensive unobservable model. IEEE Trans. Ind. Electron. 2013, 61, 1335-1344. [CrossRef]

27. Weng, C.; Sun, J.; Peng, H. A unified open-circuit-voltage model of lithium-ion batteries for state-of-charge estimation and state-of-health monitoring. J. Power Sources 2014, 258, 228-237. [CrossRef]

28. Pei, L.; Lu, R.; Zhu, C. Relaxation model of the open-circuit voltage for state-of-charge estimation in lithium-ion batteries. Iet Electr. Syst. Transp. 2013, 3, 112-117. [CrossRef]

29. Lin, C.; Yu, Q.; Xiong, R.; Wang, L.Y. A study on the impact of open circuit voltage tests on state of charge estimation for lithium-ion batteries. Appl. Energy 2017, 205, 892-902. [CrossRef]

30. Wentao, W.; Dayang, Z.; Wang, L. Systems and Methods for Battery Management. U.S. Patent 20190148951A1, 16 May 2019.

31. Tkachenko, O.; Sherstyuk, M. Battery Charging with Charging Parameters Sweep. U.S. Patent 20190081486A1, 14 March 2019. 
32. Farmann, A.; Sauer, D.U. A study on the dependency of the open-circuit voltage on temperature and actual aging state of lithium-ion batteries. J. Power Sources 2017, 347, 1-13. [CrossRef]

33. Zhang, B.; Lu, C.; Liu, J. Combination Algorithm for State of Charge Estimation. In Proceedings of the 2013 International Conference on Communication Systems and Network Technologies, Gwalior, India, 6-8 April 2013.

34. Ghantous, D.; Berkowitz, F.; Maluf, N. Method and Circuitry to Calculate the State of Charge of a Battery/Cell. U.S. Patent 8791669B2, 29 July 2014.

35. Li, X.; Choe, S.-Y. State-of-charge (SOC) estimation based on a reduced order electrochemical thermal model and extended Kalman filter. In Proceedings of the 2013 American Control Conference, Washington, DC, USA, 17-19 June 2013.

36. Ng, K.S.; Moo, C.-S.; Chen, Y.-P.; Hsieh, Y.-C. Enhanced coulomb counting method for estimating state-of-charge and state-of-health of lithium-ion batteries. Appl. Energy 2009, 86, 1506-1511. [CrossRef]

37. Wenzel, M.J.; Drees, K.H.; Elbsat, M.N. Electrical Energy Storage System with Variable State-of-Charge Frequency Response Optimization. U.S. Patent 10186889B2, 22 January 2019.

38. Liu, G.; YANG, F.; Zhang, W. Storage battery state of charge (SOC) Estimation Method Based on Extended Kalman Filtering (EKF). CN105093128A, 25 November 2015.

39. Xu, J.; Ying, Z.; Yu, Z.; Jiuhe, C. Lithium-Ion Battery SOC Estimation Based on Ahh-total Integration Method. Appl. Technol. 2018, 18, 9-11.

40. Tingting, Y.; Jie, Z.; Linkai, Z.; Yuhua, Z. SOC Estimation and Simulation of Lithium Battery Based on Improved Ampere-hour Integral Method. Energy Sav. New Energy 2018, 6, 58-60.

41. Kim, J.; Shin, J.; Jeon, C.; Cho, B. High accuracy state-of-charge estimation of Li-Ion battery pack based on screening process. In Proceedings of the 2011 Twenty-Sixth Annual IEEE Applied Power Electronics Conference and Exposition (APEC), Fort Worth, TX, USA, 6-11 March 2011.

42. Windarko, N.A.; Choi, J.; Chung, G.-B. SOC estimation of LiPB batteries using extended Kalman filter based on high accuracy electrical model. In Proceedings of the 8th International Conference on Power Electronics-ECCE Asia, Jeju, Korea, 30 May-3 June 2011.

43. Yang, W.-R.; Zhu, S.-F.; Chen, Y.; Zhu, J.-B.; Xue, L.-S.; Center, M.R. SOC estimation of lithium-ion battery based on improved ampere-hour integral method. Chin. J. Power Sources 2018, 6, 183-184.

44. Chen, L.; Lü, Z.; Lin, W.; Li, J.; Pan, H. A new state-of-health estimation method for lithium-ion batteries through the intrinsic relationship between ohmic internal resistance and capacity. Measurement 2018, 116, 586-595. [CrossRef]

45. Perkins, W.P.; Miller, K.J.; Martin, D.R.; Burke, S.R. Battery State of Charge Target Based on Predicted Regenerative Energy. U.S. Patent 9815373B2, 14 November 2017.

46. Ping, S.; Lu, L.; Gao, M.; Dong, R.; Xuning, S.F. Combined Estimation Method for Lithium Ion Battery State of Charge, State of Health and State of Function. CN105301509A, 3 February 2016.

47. Peishan, Y.; Yin, B. Analysis of SOC Estimation Algorithm for Electric Vehicle Power Battery. Automot. Pract. Technol. 2019, 15, 15-17.

48. Hongwei, L.; Caiying, S. Methods of State of Charge Estimation of Electric Vehicle. Automot. Eng. 2017, 31-33.

49. Zhou, L. Power Battery SOC Algorithm and Its Information Modeling. Electron. Technol. Softw. Eng. 2018, 88.

50. Yanhong, Y. Estimation Method and Apparatus for State-of-Charge Value of Battery. WO2017016385A1, 2 February 2017.

51. Anand, I.; Mathur, B. State of charge estimation of lead acid batteries using neural networks. In Proceedings of the 2013 International Conference on Circuits, Power and Computing Technologies (ICCPCT), Nagercoil, India, 20-21 March 2013.

52. Chen, J.; Longhui, W.; Wu, C.; Yiheng, Z. Method for Estimating State of Charge of Battery. WO Patent 2019052015A1, 21 March 2019.

53. Lipu, M.S.H.; Hannan, M.A.; Hussain, A.; Saad, M.H.; Ayob, A.; Blaabjerg, F. State of charge estimation for lithium-ion battery using recurrent NARX neural network model based lighting search algorithm. IEEE Access 2018, 6, 28150-28161. [CrossRef]

54. Xia, B.; Cui, D.; Sun, Z.; Lao, Z.; Zhang, R.; Wang, W.; Sun, W.; Lai, Y.; Wang, M. State of charge estimation of lithium-ion batteries using optimized Levenberg-Marquardt wavelet neural network. Energy 2018, 153, 694-705. [CrossRef] 
55. Wu, B.-H.; He, J.-M. The trend analysis of China's stock market based on fractal method and BP neural network model. In Proceedings of the 2014 International Conference on Management Science \& Engineering 21th Annual Conference Proceedings, Helsinki, Finland, 17-19 August 2014.

56. Dong, C.; Wang, G. Estimation of power battery SOC based on improved BP neural network. In Proceedings of the 2014 IEEE International Conference on Mechatronics and Automation, Tianjin, China, 3-6 August 2014.

57. Sun, B.; Wang, L. The SOC estimation of NIMH battery pack for HEV based on BP neural network. In Proceedings of the 2009 International Workshop on Intelligent Systems and Applications, Wuhan, China, 23-24 May 2009.

58. Hiwa, S. Secondary Battery state-of-Charge Estimating Device and Secondary Battery State-of-Charge Estimating Method. WO Patent 2016129248A1, 18 August 2016.

59. Hannan, M.A.; Lipu, M.H.; Hussain, A.; Mohamed, A. A review of lithium-ion battery state of charge estimation and management system in electric vehicle applications: Challenges and recommendations. Renew. Sustain. Energy Rev. 2017, 78, 834-854. [CrossRef]

60. Takechi, H. State-of-Charge Calculation Device, Computer Program, and State-of-Charge Calculation Method. WO Patent 2017183241A1, 26 October 2017.

61. Zhang, X.; Wang, Y.; Yang, D.; Chen, Z. An on-line estimation of battery pack parameters and state-of-charge using dual filters based on pack model. Energy 2016, 115, 219-229. [CrossRef]

62. Liu, X.L.; Cheng, Z.M.; Yi, F.Y.; Qiu, T.Y. SOC calculation method based on extended Kalman filter of power battery for electric vehicle. In Proceedings of the 2017 12th International Conference on Intelligent Systems and Knowledge Engineering (ISKE), Nanjing, China, 24-26 November 2017.

63. Jokić, Ž.Z.; Krstajić, B. State-of-charge estimation of lithium-ion batteries using extended Kalman filter and unscented Kalman filter. In Proceedings of the 2018 23rd International Scientific-Professional Conference on Information Technology (IT), Zabljak, Montenegro, 19-24 February 2018.

64. Yang, Y.; Cui, N.; Wang, C.; Liu, M.; Gao, R. SOC estimation of lithium-ion battery based on new adaptive fading extended Kalman filter. In Proceedings of the 2017 Chinese Automation Congress (CAC), Jinan, China, 20-22 October 2017.

65. Imbomi Device and Method for Estimating State-of-Charge of Battery. WO Patent 2019050330A1, 14 March 2019.

66. Cheng, S.; Zhang, Y.; Cheng, X.-F.; Zhang, X. State estimation using physics-based equivalent circuit models of a Li-ion cell and Kalman filter. In Proceedings of the IECON 2017-43rd Annual Conference of the IEEE Industrial Electronics Society, Beijing, China, 29 October-1 November 2017.

67. Liu, C.; Liu, W.; Wang, L.; Hu, G.; Ma, L.; Ren, B. A new method of modeling and state of charge estimation of the battery. J. Power Sources 2016, 320, 1-12. [CrossRef]

68. Zou, Y.; Hu, X.; Ma, H.; Li, S.E. Combined state of charge and state of health estimation over lithium-ion battery cell cycle lifespan for electric vehicles. J. Power Sources 2015, 273, 793-803. [CrossRef]

69. Tang, S.L.I. Method and system for iteratively determining state of charge of a battery cell. EP Patent 3045925A1, 20 July 2016.

70. Jun, L.; Weiyang, W.; Sihui, G.; Feife, L. Discussion on estimation method of state of charge for power battery on electric vehicle. Automot. Pract. Technol. 2019, 12-16, 21.

71. Shichang, L.; Jin, Y. Research on SOC Estimation Algorithm of Lithium Battery Based on Iterative Kalman Particle Filter. Ind. Control Comput. 2019, 32, 104-106.

72. Jianshu, G.; Hao, L.; Mingqiang, W.; Jinglun, S.; Shujian, X. An Improved Particle Filter Algorithm for SOC Estimation of Electric Vehicle Battery. Mech. Sci. Technol. 2017, 36, 1428-1433.

73. Jun, B.; Dong, Z.; Hai-tao, C.; Sai, S. Estimation for SOC of PEV Battery Based on Artificial Immune Particle Filter. Transp. Syst. Eng. Inf. 2015, 15, 103-108.

74. Tong, S.; Klein, M.P.; Park, J.W. On-line optimization of battery open circuit voltage for improved state-of-charge and state-of-health estimation. J. Power Sources 2015, 293, 416-428. [CrossRef]

75. Kim, J.; Song, T.W.; Lim, J.W. Method and Apparatus Estimating State of Battery. EP Patent 3048694A1, 27 July 2016.

76. Suglia, R.; Pritelli, D.; Aurilio, G. Method for Estimating the Current and the State of Charge of a Battery pack or Cell, Without Direct Detection of Current under Operating Conditions. EP Patent 3410139A1, 11 December 2019. 
77. Haitao, C. Research on Estimation for SOC of PEV Li-ion Battery Based on Artificial Immune Particle Filter. Master's Thesis, Beijing Jiaotong University, Beijing, China, 2013.

78. Zhang, J.; Pan, G. Comparison and application of multiple regression and BP neural network prediction model. J. Kunming Univ. Sci. Technol. 2013, 38, 61-67.

79. Zhonghua, H. Estimation Method StudyBased on Principal Component Analysis. Master's Thesis, Harbin University of Science and Technology, Harbin, China, March 2014.

80. Wu, H.-D.; Xiao-ming, R.; Wei, N.; Chao, H. Estimating SOC of Li-ion battery by improved AH combined with neural network. Battery Bimon. 2016, 46, 16-19.

81. Weiqin, L.; Ping, T.; Xu, L.; Dewang, C. Lithium Battery SOC Prediction Based on Multiple Linear Regression Model. Comput. Meas. Control 2018, 26, 145-149.

82. Qian, Y.Y.; Xiang, Z.; Guanghui, Y.; Kegang, X.; Jiayao, Z. Accurate estimation of charge state of lithium battery base on fuzzy control. Electron. Meas. Technol. 2018, 41, 7-11.

83. Andersson, H. A System and a Method for Determining State-of-Charge of a Battery. EP Patent 3371613A1, 12 September 2018.

84. Thomas, M.-P.; Ourabah, A.-D. Method for Managing the Charge State of a Traction Battery of a Hybrid Vehicle. EP Patent 3361601A1, 15 August 2018.

85. Klass, V.; Behm, M.; Lindbergh, G. Capturing lithium-ion battery dynamics with support vector machine-based battery model. J. Power Sources 2015, 298, 92-101. [CrossRef]

86. You, K.; Park, S. Method and apparatus for estimating the state of a battery. EP Patent 3048451A1, 27 July 2016.

87. Wu, Z.; Shang, M.; Shen, D.; Qi, S. SOC estimation for batteries using MS-AUKF and neural network. J. Renew. Sustain. Energy 2019, 11, 1-10. [CrossRef]

88. XinTian, L.; Hanqi, L.; Zengfu, W.; Yao, H.; Guojian, Z. CKF estimation Li-ion battery SOC based on Drift-Ah integral method. Control Decis. 2019, 34, 535-541.

(C) 2020 by the authors. Licensee MDPI, Basel, Switzerland. This article is an open access article distributed under the terms and conditions of the Creative Commons Attribution (CC BY) license (http://creativecommons.org/licenses/by/4.0/). 